\title{
Trayectoria escolar y selección universitaria: comportamiento del ranking como factor de inclusión a la educación superior*
}

\section{School background and university selection: ranking performance as an inclusion factor to higher education}

Carlos René Rodríguez Garcés** Geraldo Padilla Fuentes***
**Director del Centro de Investigación CIDCIE y académico del Departamento de Ciencias Sociales de la Universidad del Bío-Bío, Concepción, Chile. Correo: carlosro@ubiobio.cl

***Investigador adscrito al Centro de Investigación CIDCIE. Concepción, Chile. Correo: gpadilla@ubiobio.cl

Cómo citar: Rodríguez, C.R; Padilla, G.(2016) Trayectoria escolar y selección universitaria: comportamiento del ranking como factor de inclusión a la educación superior. Sophia 12 (2): 195-206.

\section{Resumen}

El proceso de admisión universitaria en Chile vive actualmente profundos cambios en su estructura en pro de la inclusión social, al valorar el esfuerzo desplegado por el alumno de distintos sectores en su trayectoria escolar. En este escenario, el presente artículo tiene por objetivo analizar el comportamiento de los factores de selección con base a las características socioeconómicas y educativas de los postulantes, haciendo especial hincapié en el impacto que tiene la incorporación del Ranking de Notas en la diversificación del alumnado y su aporte a la inclusión. Para tal efecto y haciendo uso de bases de datos del proceso de admisión a la educación superior en Chile del año 2013, se construyen una serie de índices estadísticos descriptivos, de diferencia porcentual y de asociación. Dentro de los principales hallazgos podemos consignar que los componentes trayectoria escolar, en especial el ranking, presentan distribuciones menos sesgadas y con mayor concentración en los altos puntajes y se evidencian menos influenciados por variables sociofamiliares o económicas. El ranking, como expresión del buen desempeño escolar, el esfuerzo y dedicación al estudio por parte del alumno, compensa los indeseables sesgos de selección del mecanismo tradicional, haciendo más inclusiva la opción universitaria, efecto que dependerá de la valoración que le asigne la institución universitaria.

Palabras clave: Acceso a la educación, educación superior, eficacia selectiva,inclusión social, selección igualitaria.

*Este artículo forma parte constituyente del proyecto de investigación "El Ranking de Notas: su efectividad como mecanismo de inclusión en la educación superior y su contribución a la capacidad predictiva del proceso de selección universitaria” (DIUBB 141824 2/R) aprobado por la Dirección de Investigación de la Universidad del Bío-Bío para ser ejecutado por el Centro de Investigación CIDCIE en los años 2014-2015. 
Using databases of Admission to Higher Education in Chile in 2013, the behavior of components that have school career (NEM and Ranking) and PSU scores (Mathematics and Language) is analyzed based variables segmentation of socio applicants. But both factors in theory be aligned with the curriculum, scores report a reduced correlation between them. The aim is to explore and analyze the distribution of the scores obtained by the candidates in various selection factors based on their socioeconomic and educational characteristics, and the impact of incorporating the Ranking of Scores on diversification and inclusion of the population students annually participates in the selection process. School career components, especially Ranking establishing the relative position within their respective student accommodation have less biased and with a higher concentration toward higher scores compared to the PSU component distributions, and show less influenced by variables sociofamiliar or economic. Ranking as an expression of good school performance, effort and dedication to study by the student, compensates for unwanted selection bias doing more inclusive university choice, whose effects on the modification of the student profile selected will depend on the valuation assigned the university institution to the school career the detriment of traditional PSU component.

Keywords: higher education, access to education, equal selection, social inclusion, selective efficacy.

\section{Introducción}

En Chile desde la creación del Consejo de Rectores de las Universidades de Chile (Cruch) en el año 1954, la estandarización de los instrumentos utilizados en los procesos de postulación y selección a las instituciones que lo conforman, ha evolucionado en busca de cumplir con criterios de igualdad, equidad y justicia en la selección de estudiantes independiente de las condiciones culturales, académicas y socio-económicas de origen.

Desde la antigua Prueba de Aptitud Académica (PAA) a la actual Prueba de Selección Universitaria(PSU) que rinden los estudiantes anualmente, el cuestionamiento acerca de la validez estadística y académica de los factores que las componen ha motivado discusiones en el área de la investigación social y educativa. Siendo aún un tema controversial, se discute la real capacidad de predicción de éxito universitario que tienen estos mecanismos, validez estadística, sesgos de selectividad, la escasa valoración al esfuerzo desplegado por los estudiantes en sus trayectorias escolares, así como el hecho de estar demasiado alineadas al capital social y cultural del alumnado (Koljatic y Silva, 2010; Contreras y Macías, 2002; Redondo, Descouviéres, y Rojas, 2004; Valdivieso, Antivilo, y Barrios, 2006; García-Huidobro y Belleï, 2003; Núñez y Millán, 2002).

La Prueba de Selección Universitaria - PSU- tuvo su primera aplicación el año 2004 en sustitución a la Prueba de Aptitud Académica (PAA), antiguo sistema de admisión que se mantuvo con escasa modificación por cerca de 35 años. La PSU al igual que su predecesora consiste en una batería de test de carácter obligatorio (Matemáticas y Lenguaje) y otras de carácter optativo que el alumno rinde en razón de las exigencias del programa o carrera a la que desea postular. Estas pruebas tienen una ponderación diferenciada según institución educativa y tipo de programa, las cuales son complementadas con el rendimiento escolar que el alumno ha tenido en la enseñanza media, calificación que también es traducido a puntaje estándar con base en la rama educativa (Humanista Científica Diurna, Técnico Profesional y Humanista Científica Nocturna). En consecuencia los procesos de admisión universitaria en Chile estarían estructurados con base en dos factores o dimensiones: puntaje PSU y trayectoria escolar.

La Prueba de Selección Universitaria a diferencia de la PAA estaría más alineada con el currículum definido en el Nuevo Marco para la Enseñanza Media y focalizado a los contenidos mínimos obligatorios, midiendo en consecuencia, en lugar de aptitud, el nivel de aprovechamiento educativo que el alumno tiene del currículum de enseñanza secundaria. En consecuencia su aplicación instalaría una mayor equidad en el sistema de admisión dado el supuesto de homogeneidad de los contenidos por el que transitan, en teoría, todos los estudiantes con independencia del tipo de colegio, brindando además una mayor valoración del currículum de enseñanza media. Se corregiría así el desaprovechamiento que 
las pruebas basadas en habilidad como la PAA hacía de los aprendizajes en la trayectoria escolar, y el escaso reconocimiento del esfuerzo desplegado por el estudiante.

No obstante lo anterior, la discusión con la instalación de la PSU no ha estado exenta de críticas. Pérez, Ortiz y Parra (2011) exponen que este mecanismo lo que hace es aumentar la brecha entre los establecimientos municipales y particulares pagados, ya que las pruebas se basan en un currículum nacional común del cual más del $43 \%$ de los establecimientos municipales no dicta completamente. A esto se suma la relación de legitimidad que otorga a la condición socio-económica de los estudiantes. Valdivieso (2006) en el proceso de admisión 2006 encuentra una asociación directa entre ingresos familiares y puntajes obtenidos. Por otra parte, Contreras, Corbalán y Redondo (2007) concluyen en una de sus investigaciones: "En términos prácticos, (...), la PSU se constituye como un instrumento de reconocimiento educativo para quienes provienen de situaciones culturales, sociales y económicas privilegiadas, legitimando las desigualdades sociales de origen" (p. 262). Esto contradice los fines que impulsaron la creación de este mecanismo de selección.

La búsqueda por elementos correctivos al sistema de admisión vía PSU a fin de dotarlo de una mayor equidad e inclusión social, implicó centrar la mirada en la trayectoria escolar que el alumno tenía en la educación secundaria. Así, la alineación de la prueba con los contenidos del currículum de enseñanza media se complementa con la valoración que hace el sistema de admisión a la trayectoria escolar del estudiante, premiando su esfuerzo y dedicación con su proceso de enseñanza-aprendizaje, amparado en el supuesto de que el talento académico está homogéneamente distribuido en las diferentes capas sociales y que simplemente el sistema educativo no ha sido capaz de compensar las diferencias socioculturales existentes en el entorno familiar inmediato de los alumnos.

Es así como el sistema de admisión universitario estableció un procedimiento de valoración de la trayectoria escolar, permitiendo a las universidades aumentar la ponderación a las calificaciones de enseñanza media (NEM), e incorpora desde el 2013 el Ranking como segundo elemento dentro de esta dimensión. Así este elemento, expresión de la posición relativa del alumno en su establecimiento dentro de la generación de egreso y tres generaciones anteriores con base en su promedio de notas, pasó a ser un factor más de ponderación del puntaje acumulado con el que los estudiantes postulan a las distintas universidades vinculadas al CRUCH. En la actualidad la valoración asignada al Ranking de Notas por las universidades no puede ser menor de un $10 \%$ ni mayor a un $40 \%$, por lo que la trayectoria escolar en su conjunto (NEM y Ranking) transita, dependiendo de la institución y programa, entre un $20 \%$ y un $50 \%$, ponderaciones que distan significativamente de las que asignaba el sistema previo al 2013 (Larroucau, Ríos y Mizala, 2013).

La incorporación de las notas de la enseñanza media así como el Ranking de rendimiento que tiene un alumno en su respectivo colegio ha sido, no obstante sus detractores, ampliamente defendida por la literatura tanto a nivel nacional como internacional por demostrar ser válido predictor de rendimiento académico futuro y dar reconocimiento a la trayectoria escolar previa, aspecto de preminente importancia asignada en países desarrollados (Bravo y Manzi, 2003). Al respecto, Cliffordson y Askling, (2006) en un estudio de los mecanismos de selección educacional superior en Suecia exponen que el ingreso a la educación superior vía puntaje obtenido en pruebas estandarizadas contradice los objetivos de inclusión social, educativa y nacional, por su tendencia a favorecer estudiantes que cumplen ciertas condiciones socio-económicas y culturales, además de exponer que la admisión vía mejores calificaciones en la educación secundaria cumple tanto con los criterios de inclusión como de excelencia académica a futuro.

Otros autores como Betts y Morel (1999), Gil, Paredes y Sánchez (2013) y Medina, Aguirre y Luengo (2014) exponen argumentos similares sobre la alta capacidad predictiva de las calificaciones de educación secundaria, las cuales están menos condicionadas a componentes socio-económicas o culturales de origen del estudiante, en comparación con las pruebas estandarizadas utilizadas en el proceso de selección universitaria. 
Sin embargo, el reconocimiento a la trayectoria escolar vía NEM es cuestionable por el hecho de que no todos los establecimientos tienen la misma política de asignación de calificaciones, ni en cantidad ni contenidos evaluados (Díaz, Himmel y Maltes, 1990; Contreras, Gallegos y Meneses, 2009; Prieto y Contreras, 2008; Rodríguez y Jarpa, 2015). Discusiones y evidencias de esta naturaleza conminaron a buscar un nuevo factor de trayectoria escolar más escindido de las deficiencias atribuidas a las calificaciones de enseñanza media. El Ranking a nivel internacional es considerado como un criterio de selección válido para lograr fines de inclusión y excelencia en la educación superior. En países como EEUU graduarse de secundaria entre el top 10 y $12 \%$ de la generación de egreso es vital para postularse a universidades estatales como la de Texas o California (Contreras, Gallegos, y Meneses, 2009). En el contexto nacional, Meneses y Blanco (2006) demostraron que para los alumnos de la Universidad Católica de Chile ingresados en los años 2003 y 2004 estar en el 10\% superior de los alumnos de su colegio equivale al menos a 28 puntos en el puntaje de ingreso PSU si se compara con el rendimiento académico del alumno en primer año. Por su parte Neilson y Grau (2005) de la Facultad de Economía de la Universidad de Chile concluyen que el puesto relativo del alumno en su colegio es mejor indicador que una diferencia de hasta 10 puntos en la PSU. Por ultimo, Contreras, Gallegos y Meneses (2009) exponen que el Ranking es un componente que mejora los aspectos de equidad del proceso sin afectar a los demás factores de selección, favoreciendo a segmentos sociales históricamente relegados.

En consecuencia, este artículo se enmarca dentro de la nueva discusión que a nivel nacional suscitan los recientes ajustes pro inclusión del sistema de admisión universitaria. Explora la distribución de los puntajes de los alumnos que rinden la PSU en las distintas pruebas y los puntajes asignados por trayectoria escolar, sea con ocasión de sus calificaciones obtenidas en la enseñanza secundaria (NEM) o ranking de rendimiento dentro de su unidad escolar. Análisis controlado con base en los diferentes atributos de segmentación socioeconómica y educativa para hacer hincapié en el eventual diferencial comportamiento que tienen los componentes de Trayectoria Escolar. Busca evidenciar como la mayor ponderación de factores distintos a la PSU posibilitarían aproximarse a una política de acceso a la educación superior selectiva menos restrictiva por componentes de capital sociocultural, superando así los sesgos del antiguo sistema de admisión.

\section{Materiales y métodos}

\section{Diseño}

La investigación hace uso de la base de datos del Proceso de Admisión a la Educación Superior del año 2013 proporcionada por el Departamento de Evaluación, Medición y Registro Educacional (Demre) quien tiene por función administrar el Sistema Único de Admisión a la universidad vía PSU. Esta base de datos es oficial, de fuente secundaria y acceso público. Haciendo análisis estadísticos, se indaga en el comportamiento que evidencian los distintos factores de ponderación usados en el proceso de admisión universitaria, en específico los componentes de rendimiento PSU (puntajes en las pruebas estandarizadas de Matemáticas y Lenguaje) y los de trayectoria escolar (NEM y Ranking). Análisis que tiene por finalidad determinar los comportamientos diferenciales que evidencian los factores de ponderación, sus correlaciones y las características de segmentación socioeconómica y educativa que perfilan estos comportamientos diferenciales. Ello basado en dos supuestos: primero, tener buenos indicadores de trayectoria escolar (NEM y Ranking) no es garantía de igual desempeño en las pruebas de selección, y segundo, que la valoración de la trayectoria escolar como factor de ponderación, en especial el mejor desempeño relativo o Ranking del postulante, provocaría en lo inmediato un aumento en los puntajes de postulación de sectores históricamente más postergados, perfilándose como un mecanismo de mayor inclusión social.

\section{Muestra}

La muestra de estudios estuvo constituida por los 272.663 estudiantes que rindieron la PSU el año 2013, detallada en el Cuadro 1. En cuanto a su caracterización presenta una relativa homogeneidad en su distribución por género, con una mayor presencia de mujeres (52.5\%). Dada la naturaleza misma del proceso de admisión, participa de él población principalmente joven, donde el $62 \%$ no 
supera los 19 años de edad. Alrededor de un 30\% de los jóvenes tiene entre 20 y 23 años, quienes pueden ser estudiantes rezagados académicamente, jóvenes que vuelven a participar del proceso de selección ese año con preparación preuniversitaria o que postulan con puntajes PSU del año anterior.

En su gran mayoría son alumnos recientemente egresados de la enseñanza media y pertenecen a la rama Humanista diurna $(57.4 \%)$, la cual por definición está orientada a la continuidad de estudios superiores. Las otras ramas vinculadas a la regulación de estudios y/o preparación para el mundo del trabajo, aunque no les está vedada la participación en los procesos de admisión a la educación superior, esta tiene menor relevancia estadística. En términos de dependencia administrativa solo un tercio $(33.3 \%)$ adhiere al sistema público, con una clara hegemonía del Particular Subvencionado en el otro segmento.

Tabla 1. Caracterización de la muestra

\begin{tabular}{lc}
\hline \multicolumn{1}{c}{ Sexo } \\
\hline - Hombre & $47.5 \%$ \\
- Mujer & $52.5 \%$ \\
Edad & \\
\hline - Hasta 19 años & $61.9 \%$ \\
- Entre 20 y 23 años & $30.2 \%$ \\
- 24 años o más & $7.8 \%$ \\
Dependencia Administrativa & \\
\hline - Municipal & $33.3 \%$ \\
- Particular Subvencionado & $57.0 \%$ \\
- Particular Pagado & $9.7 \%$ \\
Rama Educacional & \\
\hline - Humanista Diurno & $57.4 \%$ \\
- Humanista Nocturno & $12.8 \%$ \\
- Técnico Profesional & $29.8 \%$ \\
\hline Total & 272.663
\end{tabular}

Fuente: DEMRE - 2013. Elaboración propia

\section{- Instrumentos y procedimiento}

La base de datos del Proceso de Admisión 2013 contiene información del alumno postulante, características de su grupo familiar, establecimiento educativo en que cursó la enseñanza media, así como los puntajes que obtuvo en las pruebas de selección universitaria de Matemáticas y Lenguaje y los puntajes asignados en escala equivalente a la trayectoria escolar (NEM y Ranking).

El factor PSU contempla los puntajes de las pruebas de Lenguaje y Matemáticas, los cuales, una vez corregido el azar, son normalizados a una escala común de promedio de 500 puntos y desviación estándar de 100 puntos, transitando en una escala que va desde 150 hasta 850 puntos.

El factor trayectoria escolar contempla el promedio de notas de la enseñanza media obtenido por el alumno (NEM) y la posición relativa del alumno dentro de su establecimiento (Ranking).

Las NEM, en cuanto a calificación, son la expresión del rendimiento escolar y aprovechamiento del currículum desarrollado por el alumno en los últimos cuatro años que corresponden a la enseñanza media en Chile. Estas calificaciones son convertidas dependiendo de la rama de enseñanza a puntuación estándar PSU para hacerlas homologables como factor de ponderación.

El Ranking es una medida de la posición relativa que ocupa el estudiante en su trayectoria escolar durante la enseñanza media y busca medir de forma más precisa su desempeño con base al contexto educativo donde desarrolla su experiencia de enseñanza-aprendizaje, tomando como referencia el rendimiento de los estudiantes de las tres últimas generaciones el mismo colegio, ello con el fin de evitar generar lógicas competitivas y de inflación de notas. El Ranking es una medida de inclusión que busca valorar la trayectoria educativa y premiar el esfuerzo desplegado por el estudiante en la enseñanza secundaria, y se basa en el supuesto que el talento académico está homogéneamente distribuido en las diferentes capas sociales y tipos de colegios.

\section{Resultados}

Al momento de comparar los puntajes obtenidos y/o alcanzados en cada uno de los principales componentes que determinan la selección universitaria, las PSU registran una distribución similar en su recorrido entre niveles o rangos de puntajes con independencia del tipo de prueba (Cuadro 2). Un tercio de la población 
se acumula bajo la barrera de los 450 puntos (32.6\% en Lenguaje y $33.5 \%$ en Matemáticas), uno de cada dos postulantes no logra superar la barrera de los 500 puntos ( $49 \%$ en Lenguaje y $51 \%$ en Matemáticas) y menos de un $4 \%$ se ubica en la cúspide de los 700 puntos con independencia del tipo de prueba. Obtener sobre 450 o 500 puntos PSU son hitos relevantes por cuanto permiten tener derecho a postular a determinadas instituciones de educación superior y programas según los niveles de selectividad de estos.

Tabla 2. Componentes de rendimiento PSU y Trayectoria Escolar según rango de puntaje asignado proceso de admisión 2013

\begin{tabular}{lcccccccc}
\hline & \multicolumn{3}{c}{ Lenguaje } & \multicolumn{2}{c}{ Matemática } & \multicolumn{2}{c}{ Nem } & \multicolumn{2}{c}{ Ranking } \\
\hline Rangos & $\%$ & $\mathrm{X}$ & $\%$ & $\mathrm{X}$ & $\%$ & $\mathrm{X}$ & $\%$ & $\mathrm{X}$ \\
Menos 450 & 32.6 & $379.5(54.6)$ & 33.5 & $381.0(56.5)$ & 20.2 & $407.3(31.2)$ & 20.2 & $407.2(31.2)$ \\
$450-499$ & 16.4 & $474.0(13.2)$ & 17.6 & $478.7(13.7)$ & 22.6 & $476.6(16.4)$ & 21.0 & $475.8(16.2)$ \\
$500-550$ & 18.5 & $523.1(14.9)$ & 16.5 & $526.3(13.5)$ & 15.2 & $526.6(11.7)$ & 13.6 & $526.8(12.4)$ \\
$551-600$ & 14.9 & $574.7(15.0)$ & 14.5 & $574.4(14.6)$ & 16.0 & $575.4(15.6)$ & 13.0 & $575.2(14.9)$ \\
$601-650$ & 9.1 & $624.5(13.7)$ & 9.2 & $623.4(13.9)$ & 11.2 & $625.5(14.2)$ & 9.8 & $625.5(14.1)$ \\
$651-700$ & 5.0 & $672.3(13.2)$ & 5.2 & $671.6(14.0)$ & 6.9 & $671.0(10.4)$ & 8.2 & $674.0(14.2)$ \\
Sobre 700 & 3.5 & $738.4(30.0)$ & 3.5 & $742.6(37.5)$ & 7.9 & $732.0(28.7)$ & 14.2 & $766.7(46.8)$ \\
\hline
\end{tabular}

Fuente: DEMRE - 2013. Elaboración propia

$\mathrm{Al}$ analizar el comportamiento que tienen los puntajes asignados a los componentes de trayectoria escolar (NEM y Ranking) se observan diferencias respecto de los factores PSU. En los puntajes con base al ranking solo una quinta parte de la población (20.2\%) está bajo los 450 puntos y alrededor del $15 \%$ sobre los 700 puntos. Diferencias menos acentuadas pero igualmente relevantes se observan en el comportamiento de los grupos extremos con base en el rendimiento escolar (NEM). Especialmente significativa es la diferencia en el segmento de mejor rendimiento (sobre 700 puntos), donde se agrupa $7.9 \%$ de la distribución.

La concentración del grupo de peor rendimiento, es decir, que obtienen menos de 450 puntos en las pruebas de lenguaje y matemáticas se reduce en un $38 \%$ y $40 \%$ respecto de igual grupo cuando se considera el Ranking. De igual manera, en el grupo sobre los 700 puntos la acumulación de frecuencia de este factor es un $300 \%$ mayor en comparación a igual segmento de rendimiento en las otras pruebas. Por otra parte, las diferencias NEM-Ranking con base en la acumulación de densidad de cada grupo son bastante reducidas, patrón de comportamiento que se tiende a diversificar conforme se accede a los grupo de mejor rendimiento, con una discrepancia constante a favor del Ranking, que en el grupo de 651 - 700 puntos el delta alcanza un 19\%, mientras en el grupo inmediatamente superior esta diferencia es de un $80 \%$.

Tabla 3. Comportamiento del Ranking con las Pruebas PSU Lenguaje y Matemáticas, según atributo de segmentación

\begin{tabular}{lllllll}
\hline & \multicolumn{3}{l}{ Dif. Rank / Matemática } & \multicolumn{3}{l}{ Dif. Rank / Lenguaje } \\
& Dif. Pje & Dif. Prom $\mathrm{r}$ Pearson & Dif. Pje & Dif. Prom & r Pearson \\
\hline Dep. Administrativa & & & & & & \\
- Municipal & $80.7^{* *}$ & .21 & $.412^{* *}$ & $78.9^{* *}$ & .21 & $.382^{* *}$ \\
- P. Subvencionado & $48.0^{* *}$ & .12 & $.484^{* *}$ & $48.3^{* *}$ & .12 & $.460^{* *}$ \\
- P. Pagado & $-10.5^{* *}$ & -.01 & $.628^{* *}$ & $2.0^{* *}$ & .01 & $.624^{* *}$ \\
Rama Educativa & & & & & & \\
\hline - HC Diurno & $30.5^{* *}$ & .08 & $.559^{* *}$ & $32.6^{* *}$ & .08 & $.533^{* *}$ \\
- Téc. Profesional & $94.6^{* *}$ & .24 & $.339^{* *}$ & $92.7^{* *}$ & .24 & $.329^{* *}$ \\
- HC Nocturno & $101.5^{* *}$ & .30 & $.126^{* *}$ & $87.6^{* *}$ & .26 & $.164^{* *}$ \\
Nivel socio-económico & & & & & & \\
\hline - Bajo & $123.1^{* *}$ & .33 & $.347^{* *}$ & $22.4^{* *}$ & .33 & $.345^{* *}$ \\
- Medio bajo & $80.5^{* *}$ & .20 & $.425^{* *}$ & $76.8^{* *}$ & .19 & $.399^{* *}$ \\
- Medio & $35.8^{* *}$ & .09 & $.520^{* *}$ & $36.3^{* *}$ & .09 & $.491^{* *}$ \\
- Medio alto & $3.9^{* *}$ & .02 & $.574^{* *}$ & $7.5^{* *}$ & .02 & $.546^{* *}$ \\
- Alto & $-9.9^{* *}$ & -.01 & $.629^{* *}$ & $2.4^{* *}$ & .01 & $.617^{* *}$ \\
Educación padres & & & & & & \\
\hline - Hasta enseñanza básica & $114.5^{* *}$ & .30 & $.295^{* *}$ & $108.4^{* *}$ & .30 & $.286^{* *}$ \\
- Media incompleta & $86.6^{* *}$ & .22 & $.320^{* *}$ & $80.3^{* *}$ & .22 & $.327^{* *}$ \\
- Media completa & $65.0^{* *}$ & .16 & $.381^{* *}$ & $59.8^{* *}$ & .15 & $.369^{* *}$ \\
- Superior incompleta & $28.2^{* *}$ & .07 & $.408^{* *}$ & $24.1^{* *}$ & .07 & $.391^{* *}$ \\
- Superior completa & $22.0^{* *}$ & .05 & $.463^{* *}$ & $21.9^{* *}$ & .05 & $.445^{* *}$ \\
Total & 117.9 & .146 & .477 & 119.4 & .145 & .460 \\
\hline
\end{tabular}

Nota: Dif $=$ diferencias que presenta el Ranking con pruebas PSU de Lenguaje y Matemáticas. Dif. Pje = diferencias en términos absolutos entre Ranking y PSU. Dif. Prom. = diferencias relativas promedio de Ranking y PSU. $r$ Pearson $=$ correlación que presenta el Ranking en su relación con prueba PSU.

**: significancia estadística al .001

Fuente: DEMRE - 2013. Elaboración propia.

Como muestra el cuadro 3, al momento de comparar las correlaciones existentes entre las distintas pruebas PSU con el Ranking se obtienen índices moderados y de comportamiento conservador de .46 y .48 según se trate de la prueba de lenguaje o matemática. Los índices de correlación del Ranking y PSU son más elevados en los estudiantes de los colegios de administración Particular Pagada (matemática $\mathrm{r}=.63$; Lenguaje $r=.62 p<001$ ), de la rama Humanista Diurna (matemática $\mathrm{r}=.56$; Lenguaje $\mathrm{r}=.53 \mathrm{p}<001$ ), de nivel socioeconómico alto (matemática $\mathrm{r}=.63$; Lenguaje $\mathrm{r}=.62 \mathrm{p}<001)$, y con padres con educación superior completa (matemática $\mathrm{r}=.46$; Lenguaje $\mathrm{r}=.45$ $\mathrm{p}<001$ ), aunque en este último caso los diferenciales en los índices son menos elevados. 
Los colegios municipales registran una pérdida de variabilidad explicada respecto de los colegios particulares pagados de un $57 \%$ y un $63 \%$ para matemática $\left(\mathrm{r}^{2}{ }_{\text {MatM }}=.17 ; \mathrm{r}^{2}{ }_{\text {MatPP }}=.39\right)$ y lenguaje $\left(\mathrm{r}_{\text {LengM }}^{2}=.15 ; \mathrm{r}_{\text {LengPP }}^{2}=.39\right)$ respectivamente. De igual manera, en el alumnado de nivel socioeconómico bajo la variabilidad explicada Ranking-PSU evidencia una pérdida respecto del alumnado de nivel socioeconómico alto ascendiente al $70 \%$ en matemática $\left(\mathrm{r}^{2}{ }_{\text {MatNseA }}=.12 ; \mathrm{r}^{2}{ }_{\text {matNseE }}=.40\right)$ y $69 \%$ en lenguaje $\left(\mathrm{r}^{2}{ }_{\text {LengNseA }}=.12 ; \mathrm{r}_{\text {LengNseE }}^{2}=.38\right)$. Mucho más acentuadas y dramáticas son las pérdidas de variabilidad explicada de la rama educativa $\mathrm{HC}$ Nocturna $\left(\mathrm{r}_{\text {Leng }}^{2}=.03 ; \mathrm{r}^{2}{ }_{\text {Mat }}=.01\right)$ en comparación a la HC Diurna $\left(\mathrm{r}_{\text {Leng }}^{2}=.28 ; \mathrm{r}^{2}{ }_{\text {Mat }}=.31\right)$. La falta de sintonía entre las pruebas PSU y Ranking en la rama $\mathrm{HC}$ Diurna halla explicación en que tanto el currículum como las escalas evaluativas están orientadas a la nivelación de estudios y obtención de certificación y credenciales para la incorporación al mercado del trabajo, estando en consecuencia menos definida a la preparación para la continuidad de estudios.

A los estudiantes que participaron en el proceso de selección 2013, el Ranking les otorga un aumento comparado de alrededor de 80 puntos respecto al puntaje PSU (80.7 puntos en Matemática y 78.9 puntos en Lenguaje), diferencia que se traduce en términos relativos a un $21 \%$ más. Esta diferencia porcentual Ranking-PSU es de tan solo un 12\% en los colegios Particulares Subvencionados, y prácticamente nula en los Particulares Pagados (1\%). Diferencial comportamiento que se hace más ostensible al controlar por nivel socioeconómico del alumnado y nivel educativo de los padres.

El grupo socioeconómico bajo obtiene por concepto de Ranking 123 puntos más que en su puntaje PSU, lo que equivale a un $33 \%$ más y sin diferencias ostensibles entre las pruebas. Proporción que se reduce conforme se asciende en la escala de los ingresos. Se sitúa en alrededor de un $20 \%$ en el segmento medio Bajo, un $9 \%$ en el nivel medio y un $3 \%$ en el nivel medio alto. En el nivel Alto las mejoras eventuales que hace el Ranking son irrelevantes y se sitúan en $+/-1 \%$ según se compare con las pruebas de lenguaje o matemáticas. De igual forma, al momento de comparar por nivel educativo parental, para los alumnos cuyos padres tienen como máximo ocho años de escolaridad sus puntajes Ranking son en promedio un $30 \%$ superior al obtenido en las pruebas PSU, diferencias por sobre los 100 puntos que los convierte en el segundo grupo más favorecido ante una eventual ponderación del Ranking a la par de las pruebas PSU.

El sistema de selección universitaria independientemente de las ponderaciones asignadas por cada universidad a la trayectoria escolar y al rendimiento PSU, exige a los postulantes tener al menos 450 puntos promedio entre PSU Matemáticas y Lenguaje, no obstante algunas universidades pueden subir esta exigencia con base a la naturaleza del programa y su nivel de demanda. En el 2014 el 40\% de la oferta educativa de pregrado requería que el postulante tuviese no menos de 500 puntos PSU promedio. A ello se suma el hecho que, con base a los mayores niveles de demanda para ingresar a un determinado programa, en ocasiones los puntajes de corte se disparan por sobre las barreras de admisión reportadas por la universidad, haciendo de estas algo escasamente referencial al momento de postular.

Tabla 4. Estudiantes por cohorte de puntaje Ranking y PSU según categoría de segmentación (\%)

\begin{tabular}{lcccccccccc}
\hline & \multicolumn{1}{c}{ Sobre 500 } & \multicolumn{4}{c}{ Sobre 700 } \\
\hline Categorías de Segmentación & Rank & PSU & RR & Rank & PSU & RR & Rank & PSU & RR \\
Dependencia: & & & & & & & & & \\
- Municipal & 56.2 & 38.8 & 1.4 & 30.3 & 10.8 & 2.8 & 12.3 & 1.4 & 8.8 \\
- Particular Sub. & 55.8 & 52.9 & 1.1 & 30.2 & 15.1 & 2.0 & 13.2 & 1.5 & 8.8 \\
- Particular Pagado & 80.6 & 93.2 & 0.9 & 51.6 & 64.8 & 0.8 & 27.1 & 16.7 & 1.6 \\
Rama educacional & & & & & & & & & \\
- Humanista diurno & 63.2 & 66.0 & 1.0 & 35.4 & 26.9 & 1.3 & 16.0 & 4.4 & 3.6 \\
- Humanista nocturno & 50.2 & 15.9 & 3.2 & 26.9 & 1.4 & 19.2 & 11.0 & 0.1 & 110. \\
- Técnico profesional & 44.6 & 16.0 & 2.8 & 20.7 & 2.6 & 8.0 & 9.1 & 0.1 & 91.0 \\
Educación Padres & & & & & & & & & \\
- Hasta enseñanza básica & 58.5 & 24.9 & 2.3 & 32.8 & 3.8 & 8.6 & 14.4 & 0.2 & 72.0 \\
- Media Incompleta & & & & & & & & & \\
- Media Completa & 52.9 & 30.0 & 1.8 & 27.7 & 4.8 & 5.8 & 10.9 & 0.2 & 54.5 \\
- Superior Incompleta & 55.5 & 42.6 & 1.3 & 29.4 & 9.9 & 3.0 & 12.4 & 0.8 & 15.5 \\
- Superior Completa & 57.7 & 61.7 & 0.9 & 30.1 & 20.4 & 1.5 & 12.3 & 2.3 & 5.3 \\
& 68.3 & 74.8 & 0.9 & 40.3 & 37.1 & 1.1 & 19.4 & 7.2 & 2.7 \\
Nivel socio-económico & & & & & & & & & \\
- Bajo & 51.4 & 28.3 & 1.8 & 27.6 & 3.7 & 7.5 & 11.7 & 0.2 & 58.5 \\
- Medio & 58.7 & 61.0 & 1.0 & 31.5 & 16.8 & 1.9 & 13.2 & 1.4 & 9.4 \\
- Alto & 72.1 & 85.9 & 0.8 & 42.6 & 47.5 & 0.9 & 20.5 & 9.6 & 2.1 \\
\hline
\end{tabular}

Nota: los valores expresan porcentajes de los estudiantes que al interior de cada segmento pertenecen a determinada cohorte de puntaje; Particular sub= particular subvencionado; Rank = Ranking, posición relativa del alumno en su establecimiento de egreso homologado a puntaje PSU; PSU = puntaje pruebas selección universitaria RR: Riesgo Relativo, señala cuanto es más probable que un sujeto que pertenece a un determinado segmento integre una determinada cohorte de puntaje Ranking en comparación a pertenecer a igual cohorte PSU.

Fuente: DEMRE - 2013. Elaboración propia. 
Teniendo lo anterior como antecedente, el cuadro 4 analiza la distribución de los estudiantes por puntaje de cohorte tanto Ranking como Promedio PSU (Lenguaje y Matemáticas) según variables de caracterización.

En primera instancia, se observa que al considerar las pérdidas de densidad en la distribución de estudiantes por cohorte de puntaje, lograr un alto rendimiento en el colegio de egreso no es sinónimo de similar comportamiento al enfrentar las pruebas de selección universitaria, al menos no para los postulantes egresados de establecimientos financiados por el Estado (Municipal o Part. Subvencionado) y de bajo capital cultural y económico.

En el mismo cuadro se observa el diferencial comportamiento de los factores de ponderación en las distintas cohortes con base al perfil socioeducativo del estudiante. El factor PSU privilegia principalmente a los alumnos de nivel socioeconómico alto, de colegios Particular Pagados, cuyos padres tienen educación superior y pertenecen a la rama $\mathrm{HC}$ Diurna. En efecto, de los estudiantes que egresan de algún establecimiento Particular Pagado 9 de cada $10(93.2 \%)$ aseguran su participación en los procesos de postulación a universidades selectivas al obtener sobre 500 puntos PSU, mientras que de los establecimientos Municipales solo 4 de cada 10 (38.8\%) egresados obtiene igual logro.

Al comparar por nivel socioeconómico dentro de la misma cohorte y factor, estas proporciones son del $85.9 \%$ en el NSE Alto y de un $28.3 \%$ en el NSE Bajo. Conforme aumentan los puntajes de cohorte, como es lógico dado los mayores niveles de exigencia, la acumulación de densidad tiende a disminuir en todos los segmentos. Diferencias más ostensible en los grupos de menor nivel socioeconómico, de bajo nivel educativo de los padres y de colegios municipales. Así por ejemplo, mientras en los colegios Particular Pagados un $16.7 \%$ superaba la barrera de los 700 puntos PSU, en los colegios municipales esta proporción apenas alcanzaba el $1.4 \%$, cifra 11 veces menor.

Estas diferencias, si bien transversales, al analizar el comportamiento del Ranking, se atenúan significativamente. Sobre la barrera de los 500 puntos la concentración de estudiantes de colegios municipales es un $45 \%$ más que al considerar exclusivamente el rendimiento PSU. Incluso en el cohorte sobre 700 puntos mientras en el factor PSU solo un $1.4 \%$ superaba esta barrera, al considerar el Ranking la concentración de estudiantes de colegios municipales es de un $12.3 \%$, es decir 8 veces superior. Similar comportamiento se constata al analizar la acumulación de frecuencia por cohorte de puntaje del Ranking en las diferentes variables de segmentación.

Tener padres con escolaridad superior es un factor que refuerza el rendimiento de los estudiantes tanto al enfrentarse a instancias como la selección universitaria como a través de todo el proceso educacional, ya que son ellos quienes más llegan a obtener altos puntajes Ranking y PSU (6\% en PSU y $17 \%$ en Ranking).

En comparación, los estudiantes con padres cuya escolaridad no supera la enseñanza media completa, el $63 \%$ no traspasa la barrera de los 500 puntos PSU promedio y menos del $1 \%$ se posiciona sobre 700 puntos en el mismo factor. El efecto del capital cultural tiende a ser corregido por el Ranking, el cual muestra mayor estabilidad entre las categorías educativas a medida que las cohortes de puntaje aumentan. De los estudiantes cuyos padres tienen 12 o menos años de escolaridad, un 56\% se posiciona en la cohorte sobre 500 puntos Ranking y un 13\% sobre los 700 puntos en igual factor.

La posibilidad de que un alumno de establecimiento financiado por el Estado (Municipal o Particular Subvencionado) pertenezca a la cohorte "sobre 700" Ranking es 8.8 veces superior a la de pertenecer a igual cohorte PSU (sobre 700 puntos); cuando pertenecen a nivel socioeconómico bajo esta posibilidad es 58 veces y 72 cuando sus padres no han completado la enseñanza básica. Como contraparte y en igual cohorte de alto rendimiento (sobre 700), la probabilidad Ranking/PSU es de 1.6 en alumnos de Colegios Particular Pagado, 2.1 en NSE Alto y 2.7 en familias con padres con educación superior completa.

\section{Discusión de resultados}

A nivel general las puntuaciones promedio obtenidas por los alumnos con base al ranking tienden a ser superiores a las de los otros componentes o factores contemplados en el puntaje de admisión. Aunque en 
estricto rigor metodológico estas diferencias no dan cuenta de niveles de aprovechamiento educativo o rendimiento diferenciado, por cuanto las pruebas PSU lenguaje y matemáticas son estandarizadas y el ranking no lo es, la mayor puntuación del ranking si manifiesta sus efectos como factor de ponderación en el puntaje final con que postulan los alumnos a distintas universidades.

De manera complementaria, al comparar los grupos y segmentos con base en el rendimiento en los distintos componentes o factores que se consignan en los procesos de admisión (rendimiento PSU y trayectoria escolar), se constatan grandes diferencias en la acumulación de densidad de cada segmento o categoría de rango, en especial, en los grupos de mayor rendimiento, siendo las menos acentuadas las establecidas entre los componentes de la Trayectoria Escolar.

Con base en el análisis del comportamiento de los factores de admisión se desprenden dos supuestos. Primero, la obtención de un buen Ranking no garantiza necesariamente un buen desempeño en las pruebas de selección universitaria, así como tampoco lo hace la obtención de un buen NEM o rendimiento académico, argumentación que está en la línea de lo planteado por Contreras, Gallegos y Meneses (2009), Larrocau, Ríos y Mizala (2013), y Medina, Aguirre y Luengo (2014). Segundo, el mejor desempeño relativo obtenido en el Ranking provocaría en lo inmediato un aumento en los puntajes de postulación y una diferenciación de las universidades respecto a la valoración que le otorgan como factor de ponderación incluido con tales fines.

No obstante las restricciones que presentan los índices de correlación en el espacio bivariante, sus índices dan cuenta de la baja capacidad predictiva que tiene el Ranking al momento de estimar el rendimiento en las pruebas PSU, por lo que obtener un promedio alto y buen posicionamiento en un determinado colegio no es suficiente garantía para augurar buenos resultados en los procesos de selección universitaria. Situación que se acentúa conforme disminuye el nivel socioeconómico del alumnado, se pertenece a establecimientos municipales o egresa de la rama de educación nocturna.
El desalineado comportamiento que tienen los componentes de Trayectoria Escolar con los test PSU es reflejo de la segmentación socio-educativa, de los diferenciales niveles de exigencia y la falta de homogeneidad en la política evaluativa de los distintos establecimientos. El Ranking y NEM son expresión del nivel de aprovechamiento que el estudiante hace del currículum en su trayectoria escolar. En consecuencia, se espera que los puntajes obtenidos por estos conceptos correlacionen con mayor intensidad con los alcanzados en los test PSU, pruebas estandarizadas que se formulan en observancia al mismo currículum.

Si bien la obtención de un buen Ranking no es garantía de similar desempeño en las pruebas PSU, su inclusión como mecanismo de ponderación aportaría una mayor equidad al sistema en la medida que su comportamiento favorece a grupos más postergados y que históricamente han evidenciado dificultades para acceder a instituciones de educación superior de carácter selectivo cuando el sistema de selección solo ha contemplado el rendimiento PSU, asignando una irrelevante valoración a la trayectoria escolar, con una ponderación que no superaba en la gran mayoría de los casos el 10\%. En consistencia con lo expuesto las diferencias promedios de los puntajes PSU lenguaje y matemática con base en el Ranking muestran un comportamiento acorde con lo que motivó su inclusión como factor de ponderación ya que aumenta los puntajes de la gran mayoría de los estudiantes, beneficios que se acentúan en sectores estratégicos.

El comportamiento diferencial que tiene el Ranking en relación al nivel de logro PSU se traduce en lo inmediato en una distorsión en la ponderación de los factores de selección que adoptan las universidades, en consideración a distintos criterios. Las hay quienes, basadas en juicios de mayor inclusión social, asignan alta ponderación a la trayectoria escolar (NEM y Ranking) buscando mejorar los puntajes de los estudiantes, máxime cuando estos pertenecen a grupos a los cuales la obtención de un buen puntaje PSU les ha sido históricamente complicada. Nos referimos aquí a alumnos primera generación, de bajo niveles de renta, menor capital cultural y reducido capital humano familiar acumulado. 
Por otra parte, existe un contingente relevante de universidades que transitan en los mínimos legales establecidos de valoración de la trayectoria escolar, Ranking incluido. En el año 2014 en un tercio de la oferta educativa de pregrado esta dimensión alcanzaba, repartido en sus dos factores, una ponderación máxima de $30 \%$. En estas instituciones se apela a razones de justicia amparado en el hecho que se debe premiar el esfuerzo que un estudiante realiza para alcanzar un buen puntaje PSU, sumado a la desconfianza de las políticas evaluativas que desarrollan los establecimientos que muchas veces se distancian del rigor métrico y objetivo, haciendo subir artificiosamente las calificaciones de sus propios alumnos de enseñanza media con propósitos espurios.

El capital cultural de los estudiantes, expresado en la escolaridad de sus padres, es un factor difícil de contrapesar al rendir la PSU. Aunque este capital familiar acumulado se correlaciona con el nivel de ingreso y el tipo de experiencia educativa recibida, los datos informan que conforme aumenta la escolaridad de los padres también lo hacen los puntajes PSU. Al observar las diferencias entre los niveles socioeconómicos de los estudiantes con base en el Ranking, estas se hacen menos ostensibles. En concreto las cohortes de puntaje por este factor acumulan mayor densidad en los grupos más vulnerables, contrario a lo que sucede en las cohortes PSU. Así mismo, las brechas entre grupos se atenúan.

El Ranking como medida de acción afirmativa favorece a los grupos más vulnerables y que han tenido una experiencia educativa segmentada en términos de calidad y pertinencia. Lo expuesto se ratifica al comparar los índices de Riesgo Relativo que tienen los diferentes segmentos socioeducativos analizados. En consecuencia, se puede establecer que la consideración del Ranking permitiría una mejora en los puntajes de postulación de estudiantes que provienen de los niveles socioeconómicos más precarios, cuyos padres presentan baja escolaridad y provienen de colegios públicos, reduciendo además las brechas existentes entre los grupos de atributos socioeducativos diferenciados, dando la posibilidad de corregir el sesgo selectivo que tienen las pruebas estandarizadas que correlacionan con indeseada frecuencia con el capital cultural y económico del alumno.

\section{Conclusiones}

El abordaje a la discusión acerca del proceso de selección universitaria y la doble dimensionalidad de los factores que lo componen (rendimiento PSU y Trayectoria Escolar), nos ha llevado a través del análisis del proceso de selección universitaria 2013, a buscar cuales son los cambios que produjo la inclusión del Ranking de Notas como factor de ponderación ese año, el comportamiento de los puntajes obtenidos por los estudiantes en este factor, las características de quienes serían más o menos favorecidos con esta medida, su distribución en los estudiantes según cohortes de puntajes y cuáles son las eventuales diferencias que tienen con la dimensión PSU y su símil, las NEM.

Como resultado de lo anterior, los hallazgos más relevantes fueron:

Los puntajes obtenidos por los postulantes en su trayectoria escolar (NEM y Ranking) son un factor de ponderación que muestra una menor relación con determinados atributos que se pueden considerar de segmentación social (NSE Alto, Capital Humano Familiar Acumulado Alto, Dependencia Particular Pagada), en comparación con los factores PSU. Esto provocaría en lo inmediato un aumento en los puntajes de postulación de los estudiantes que impacta de mayor forma en sectores estratégicos, de alta vulnerabilidad social y bajo capital cultural y económico, lo que mejora los criterios de selección del proceso al permitir el ingreso de estudiantes provenientes de tramos históricamente postergados a la educación superior de carácter selectivo.

En la dimensión de Trayectoria Escolar, la ponderación del desempeño relativo o Ranking del postulante muestra mayores beneficios que las NEM. Como este puntaje tiene su base en las notas del alumno, no puede ser inferior, lo que provoca un alza en la proporción de estudiantes con mejor puntaje Ranking que NEM, reforzando lógicamente lo expuesto en el punto anterior.

Los potenciales beneficios que entrega el Ranking tienen como base su baja relación con los atributos de origen de los estudiantes, contrario a la intensidad que mantiene la dimensión PSU con dicha característica. $\mathrm{Al}$ analizar el comportamiento y la distribución de los 
puntajes obtenidos en las pruebas, damos cuenta de que la obtención de un buen NEM o Ranking no es garantía o preámbulo de un buen desempeño PSU, ya que las relaciones entre ambas dimensiones no son regulares. Sus diferencias en la acumulación de estudiantes se magnifican a medida que aumenta el nivel de logro alcanzado, evidenciando además comportamientos irregulares según determinados criterios de control.

El aumento en los puntajes de la Trayectoria Escolar y por consiguiente de los ponderados con que postulan los estudiantes a las diferentes universidades, presenta para estas instituciones el desafío de la valoración a los cambios implementados en el mecanismo de selección que expresan y a la ponderación que otorgan a los diferentes factores. La validación que le den a la dimensión de la trayectoria escolar otorgándole una alta ponderación conllevará la atracción de estudiantes con habilidades distintas de aquellos seleccionados tras alcanzar altos puntajes en la dimensión PSU. Una mayor valoración institucional a la trayectoria educativa como factor de ponderación hará más inclusiva a la educación universitaria sin alterar negativamente la capacidad predictiva de la PSU, pues la evidencia muestra que los estudiantes top de sus colegios de egreso tienen mejor rendimiento universitario que sus pares no top, no obstante, las posibilidades de ingreso que tienen estos estudiantes a universidades y carreras altamente selectivas que asignan alta ponderación a la dimensión PSU son bajas, en especial cuando estos buenos estudiantes provienen de colegios de financiamiento público, de situación vulnerable y de familias de bajo capital cultural.

Sin duda, la configuración de las ofertas de pregrado y programas de estudio de las diferentes universidades que se nutren del proceso de selección será decisiva para las futuras discusiones acerca de los mecanismos de selección universitaria, además de reflejar la decisión que tomen las universidades respecto al dilema de cuáles son los mejores factores predictores de buen desempeño universitario y por consiguiente de excelencia académica. La pertinencia de estas modificaciones dividirá a las universidades y abrirá nuevos diálogos acerca del porqué y de la intencionalidad que los motiva.

\section{Referencias bibliográficas}

Betts, J. y Morel, D. (1999). The Determinants of undergraduate grade point average. The relative importance of family background high school recources, and peer group effects. Journal of Human Resources. 268-293.

Bravo, D. y Manzi, J. (2003). El SIES, la equidad y la elevación de los aprendizajes [The SIES, equity and the improvement of learning]. Retrieved April 20.

Contreras, D. y Macías, V. (2002). Desigualdad educacional en Chile: geografía y dependencia. Cuadernos de economía, 39(118), 395-421.

Contreras, D., Gallegos, S. y Meneses, F. (2009). Determinantes del desempeño universitario: ¿ importa la habilidad relativa? Revista Calidad en la Educación, 17-48.

Contreras, M., Corbalán, F., y Redondo, J. (2007). Cuando la suerte está hechada: Estudio cuantitativo de los factores asociados al rendimiento en la PSU. Revista Electrónica Iberoamericana sobre Calidad, Eficacia y Cambio en Educación. 5(5), 259-263.

Díaz, E., Himmel, E. y Maltes, S. (1990). Evolución histórica del sistema de selección a las Universidades Chilenas, 1967-1989. En Lemaitre, María José (Editora), La educación superior en Chile: un sistema de transición. Santiago de Chile: Corporación de Promoción Universitaria (CPU), Colección Foro de la Educación Superior.

García Huidobro, J. y Belleï, C. (2003). Desigualdad educativa en Chile. Universidad Alberto Hurtado, Escuela de Educación.

Gil, F., Paredes, R. y Sánchez, I. (2013). El ranking de las notas: inclusión con excelencia. Centro de políticas públicas de la UC.60.

Koljatic, M. y Silva, M. (2010). Algunas reflexiones a siete años de la implementación de la PSU. Estudios públicos, (120), 125-146. 
Larroucau, T., Ríos, I., y Mizala, A. (2013). Efectos de la incorporación del ranking de notas en la selección universitaria. Santiago: Demre.

Medina, A., Aguirre, M. y Luengo, L. (2014). Predictibilidad de las notas de enseñanza media según establecimiento de origen sobre el rendimiento académico en estudiantes de Odontología. Educación Médica Superior, 28(1), 65-73.

Meneses, F. y Blanco, C. (2006). La posición en el Ranking y el puntaje de ingreso en la PUC: implicancias prácticas. Santiago: Departamento de Estudio Mineduc.

Núñez, J. y Millán, I. (2002). ¿ Pueden mejorar su PAA los alumnos de escasos recursos?: evidencia experimental. Cuadernos de economía, 39(116), 5-25.

Pérez, C., Ortiz, L., \& Parra, P. (2011). Prueba de Selección Universitaria, rendimiento en enseñanza media y variables cognitivo-actitudinales en alumnos de Medicina. Revista de Educación, Ciencia y Salud, 120-127
Prieto, M. y Contreras, G. (2008). Las concepciones que orientan las prácticas evaluativas de los profesores: un problema a develar. Estudios pedagógicos (Valdivia), 34(2), 245-262.

Redondo, J., Descouvières, C. y Rojas, K. (2001). Equidad y calidad de la educación en Chile: Reflexiones e investigaciones de eficiencia de la educación obligatoria (1990-2001). Universidad de Chile, Facultad Ciencias Sociales, Vicerrectoría Investigación.

Rodríguez, C. y Jarpa, C.G. (2015). Capacidad predictiva de las notas en enseñanza media sobre el rendimiento en pruebas de selección universitaria: el caso chileno. Aula Abierta, (43) 61-68.

Valdivieso, P. Antivilo, A. y Barrios, J. (2006). Caracterización educacional y sociodemográfica de los estudiantes que rinden la psu, postulan y se matriculan en las universidades reunidas en el consejo de Rectores. Revista Calidad en la Educación, (24). 